\title{
Large Scale Antenna Selection and Precoding for Interference Exploitation
}

\author{
Pierluigi V. Amadori, Student Member, IEEE, and Christos Masouros, Senior Member, IEEE
}

\begin{abstract}
We propose several low-complexity Transmit Antenna Selection (TAS) and precoding schemes for Massive multi-input multi-output (M-MIMO). It is well established that large antenna arrays in M-MIMO lead to particularly high hardware overheads as they require an equally large number of radio-frequency chains, and antenna selection is envisaged as a solution to reducing this hardware complexity. Accordingly, in the proposed schemes, both hardware and computational complexity of M-MIMO systems are addressed by jointly optimizing TAS and precoding. We first introduce a mixed-integer programming approach that simultaneously identifies the transmitting antennas subset and solves the precoding problem, by employing a unified metric based on Constructive Interference (CI) concept. We then propose three sub-optimal techniques that allow a reduction of the computational complexity required to solve the joint optimization. Our analyses and results prove that the proposed joint TAS and precoding schemes based on CI exploitation are able to outperform the state-of-the-art, while providing a favorable performance-complexity trade-off.
\end{abstract}

Index Terms-Massive MIMO, Multiuser MIMO, Antenna selection, Interference optimization

\section{INTRODUCTION}

In recent years, the research community has considered M-MIMO as one of the most promising technologies for future wireless communication systems [1], [2]. The seminal work in [2] showed that MIMO systems with very large arrays (VLAs) are able to achieve extremely high throughputs by exploiting the innate high degrees of freedom offered by a large number of antennas at the transmitter. In addition, it was proven that infinitely large arrays lead to very favorable propagation effects, which make simple linear precoding techniques asymptotically optimal [1]. Further works showed that the key concepts behind M-MIMO can be also applied to multiuser scenarios by equipping base stations (BS) with VLAs that greatly out-scale the number of served users [3], allowing to perform secure, robust and energy-efficient communications with increased throughputs.

Manuscript received October 25, 2016; revised March 14, 2017 and May 22, 2017; accepted June 18, 2017. The associate editor coordinating the review of this manuscript and approving it for publication was Dr. Zhaoyang Zhang.

The authors are with Department of Electronic \& Electrical Engineering, University College London,Torrington Place,London WC1E7JE,UK

This work was supported by the Royal Academy of Engineering, UK and the Engineering and Physical Sciences Research Council (EPSRC) project EP/M014150/1.
While the theoretical benefits of M-MIMO systems are undoubtedly very appealing, their practical implementation are just as equally challenging for both hardware requirements and signal processing. In fact, since each radiating element is connected to a radio-frequency (RF) chain, a BS equipped with a VLA requires an equally large number of amplifiers, analog-to-digital converters and mixers. These considerations have brought to many array configurations, such as cylindrical or planar arrays, which are mostly characterized by small active units [3] to respect cost and space constraints. Toward this end, recent works [4]-[7] have also investigated the possibility of increasing the number of array elements in fixed physical spaces by exploiting the deriving transmit mutual coupling at the BS.

In the past years, many works [8]-[12] showed that the hardware complexity deriving from the use of multiple antennas can be tackled by means of antenna selection, either at the transmitter or at the receiver side. In antenna selection systems, only a subset of the available antennas is actually used in transmission or reception [13], allowing for a reduction in the RF chains employed, while also benefiting from augmented antenna diversity. First approaches were based on exhaustive search [11], [14], however this proves to be a prohibitively costly approach as the numbers of transmitter or receiver increase [10]. Toward this end, the authors in [10] introduced a selection algorithm based on the minimization of the capacity losses deriving from the usage of a subset of antennas. Such approach proved to be cost efficient for low-dimensional MU-MIMO [10], especially when compared with exhaustive searches [12], [15], however its direct application to M-MIMO was shown to be nearly prohibitive [16]. Since a direct application of MIMO TAS techniques to massive systems can be impractical in terms of computational costs, recent works [17]-[19] focused on TAS algorithms precisely designed for M-MIMO systems ${ }^{1}$. More specifically, the work in [20] presents a TAS study under the

\footnotetext{
${ }^{1}$ While TAS reduces the beneficial effects of M-MIMO with a full transmission (i.e., with one RF chain per radiating element), such as the channel hardening or the asymptotic optimality of simple linear precoding, it is also true that TAS is able to exploit the vastly enhanced spatial selectivity of VLAs. In fact, in TAS-based systems, a reduced number of RF chains (i.e., one per user) can serve a larger number of antennas, while improving the system performance in terms of power efficiency and hardware complexity.
} 


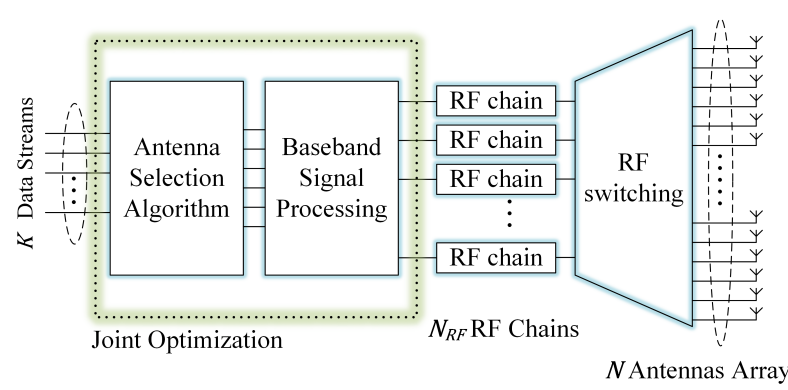

Fig. 1. Conventional TAS block diagram.

perspective of energy efficiency, while the authors in [21] study a random selection approach. Finally, [22] and [19] proposed the use of convex optimization for M-MIMO TAS systems, for a massively distributed antenna system and for channel capacity optimization respectively.

State-of-the-art TAS-based multiuser MIMO (MUMIMO) systems are known to approach selection and downlink precoding as two disjointed optimization problems [8], [9]. In fact, TAS systems from the literature are characterized by a cascade of the antenna selection and either linear or nonlinear precoding [10]-[12], [16], [23], as shown in Fig.1. This is caused by the fact that conventional TAS algorithms and precoding designs are based on different and disjointed metrics, hence leading to two separated optimization problems. In contrast with this, we propose a fully digital novel transmission approach where both TAS and precoding can be jointly performed by optimizing a single performance metric, shared by both techniques ${ }^{2}$. Joint TAS and precoding optimization offers the unique opportunity to maximize interference exploitation benefits, as TAS provides the extra degrees of freedom in the optimization. More specifically, the proposed schemes are based on the optimization of a CIbased metric [28], [29], where the resulting multiuser interference (MUI) is manipulated in order to constructively align to the useful received signal. The deriving optimization is a Mixed-Integer Programming (MIP) problem and can be efficiently solved by commercial optimization solvers. In addition to the proposed MIP-based approach, we present three different heuristic solutions to the optimization problem and their performances are analyzed. More specifically, the proposed schemes employ a novel low-complexity TAS-precoding algorithm to retain the maximum benefits of Constructive Interference (CI) [28][37] in a MU-M-MIMO scenario. It has been established in [28], [29] that MUI can be beneficial for downlink transmission performances both in terms of signal detection and power efficiency, as it allows to increase the received power of the desired signal. Given the fact that interference is data dependent, the transmitter is able to predict MUI and can use this knowledge to manipulate

\footnotetext{
${ }^{2}$ While the proposed algorithms have been specifically tailored for a fully digital system, they could be directly applied to hybrid digitalanalog precoding schemes [24]-[27], where a reduced number of RF chains is able to serve a larger number of beams.
}

it and capitalize on it. More specifically, works in [28], [29] showed that the transmitted signal can be effectively precoded in order to rotate the destructive component of interference to align the interfering transmissions towards the desired signal. Finally, the authors in [38] have studied the possibility to exploit CI-based symbol-level precoding concepts to enhance the security of MU-MIMO communications by means of Directional Modulation [39].

While the proposed schemes have been specifically tailored for Phase-Shift Keying (PSK) modulation scenarios, recent works have shown that such concepts can be readily adapted to accommodate CI exploitation in Quadrature Amplitude Modulation (QAM) [40], [41]. In fact, the authors in [42] have proven that the benefits of CI can extend to 16-QAM modulations by allowing the predictable interference at the BS to constructively superimpose with the desired signal at the receiver side. Analogously, [43] presented constraints and metrics for QAM and asymmetric phase-shift keying modulations, proving that the interference exploiting approach on a symbol-level precoding can strongly enhance power efficiency at the BS.

The developed algorithms introduce a novel approach to M-MIMO TAS systems and are designed to fully exploit both CI and the high diversity offered by VLAs by jointly selecting a small subset of transmitting antennas and defining the precoded signal. The joint optimization enables to fully exploit the benefits of both TAS and precoding. In fact, the proposed joint TAS-precoding allows to greatly reduce the number of RF chains at the BS, hence achieving both a significant mitigation of hardware complexity and power consumption at the transmitter side, and an important reduction of the signal processing required.

Here we list the contributions of the paper:

- We introduce a novel transmission scheme for multiuser M-MIMO scenarios based on concepts of CI exploitation that jointly performs TAS and precoding;

- We define a MIP-based and three low-complexity heuristic approaches to efficiently solve the proposed optimization problem. We further evaluate the optimality of these heuristic approaches;

- We study the effects of imperfect Channel-State Information (CSI) over the presented metrics and derive a CSI-robust approach for the proposed techniques;

- We evaluate the performances obtained by the proposed schemes in terms of Symbol Error Rate (SER), Capacity and an energy efficiency metric that combines throughput with system power requirements.

The rest of the paper is organized as follows: Section II introduces the system model used throughout this work, CI concepts are described in Section III. The joint MIP TAS/precoding optimization problem is derived and solved in Section IV. In Section V three heuristic approaches with decreasing computational com- 
plexity are presented. The optimality of the proposed heuristic schemes is discussed in detail in Section VI. Section VII describes the benchmark techniques used in the paper, while Section VIII investigates the computational requirements of the proposed schemes. Channel uncertainty effects are analyzed in Section IX and a robust approach to the transmission optimization scheme is presented. Finally, Section $\mathrm{X}$ is dedicated to a study of the performances obtained with the proposed system, while Section XI summarizes the contributions of the paper.

Notation: We use the following notation throughout the paper. Upper case boldfaced letters identify matrices (i.e. $\mathbf{X})$, lower case boldfaced letters are used for vectors (i.e. $\mathbf{x})$, vector subindices are used to identify the columns of a matrix (i.e. $\mathbf{x}_{m}$ is the $m$-th column of $\mathbf{X}$ ), $\operatorname{tr}[\cdot]$ represents the trace of a matrix, $\operatorname{diag}(\cdot)$ identifies the diagonal of a matrix, superscripts $(\cdot)^{T},(\cdot)^{H}$ and $(\cdot)^{*}$ stand respectively for transpose, Hermitian transpose and complex conjugate.

\section{SySTEM MOdEL}

In our analyses, we investigate the application of the proposed schemes to the PSK-modulated downlink transmission in a multiuser scenario ${ }^{3}$, where the BS adopts a very large $N$-sized array to communicate with a population of $K$ single antenna users. The signals received by the $K$ users are collected in a $\mathbb{C}^{K \times 1}$ vector $\mathbf{y}$, whose $k$-th element is defined as:

$$
y_{k}=\mathbf{h}_{k}^{T} \mathbf{x}+n_{k}=\sum_{n=1}^{N} h_{n, k} x_{n}+n_{k},
$$

where $\mathbf{h}_{k}$ identifies the $\mathbb{C}^{N \times 1}$ channel vector of the $k$ th user, i.e. the channel matrix $\mathbf{H}=\left[\mathbf{h}_{1}, \ldots, \mathbf{h}_{k}, \ldots, \mathbf{h}_{K}\right]$, $\mathbf{x}$ is the $\mathbb{C}^{N \times 1}$ transmitted signal and $n_{k}$ is the $k$ th component of the $\mathbb{C}^{K \times 1}$ zero mean additive white Gaussian noise vector $\mathbf{n}$, i.e. $\mathbf{n} \sim \mathcal{C N}\left(0, N_{0}\right)$ with $N_{0}$ being the noise variance. Each $h_{n, k}$ entry of the channel matrix $\mathbf{H}$ individually represents the complex path gain between the $n$-th transmitting antenna at the BS and the $k$-th user and can be modelled as [2]

$$
h_{n, k}=\alpha_{n, k} \sqrt{\beta_{k}},
$$

where $\alpha_{n, k}$ identifies the complex fast-fading experienced by the $n$-th transmitted symbol when received by the $k$ th user and $\beta_{k}$ represents the real slow-fading coefficient of the $k$-th user. In our studies, we consider a single-cell downlink transmission scenario and model each element of the channel matrix as independent Rayleigh fading, i.e. $h_{n, k}, \forall n \in\{1, \ldots, N\}, k \in\{1, \ldots, K\}$ is a zero mean

\footnotetext{
${ }^{3}$ It is important to highlight that while the proposed schemes are specifically designed for PSK modulations, their optimization metrics could be adapted to accommodate CI exploitation to quadrature amplitude modulation schemes, as shown in [42], [43].
}

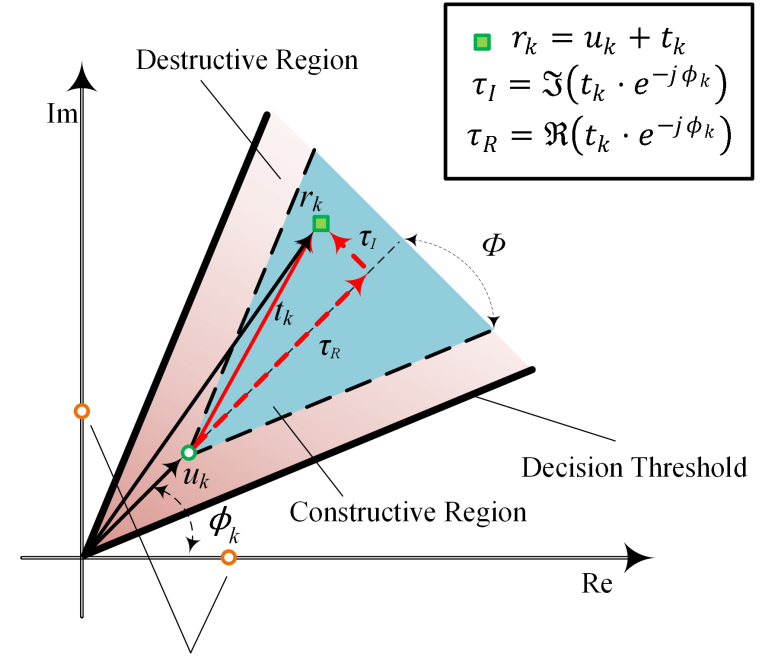

Adjacent Constellation Points

Fig. 2. Constructive Interference region for 8-PSK symbol.

independent and identically distributed complex Gaussian variable [44].

When TAS is considered, the received signal equation for the $k$-th user $\tilde{y}_{k}$ and the transmitted signal from the $n$-th antenna need to be accordingly modified as follows

$$
\tilde{y}_{k}=\sum_{n=1}^{N} h_{n, k} \tilde{x}_{n}+n_{k},
$$

where $\tilde{x}_{n}$ represents the $n$-th element of the $\mathbb{C}^{N \times 1}$ precoded transmitted signal $\tilde{\mathbf{x}}$ and whose value is null when its index corresponds to one of the deactivated antennas, i.e., $\tilde{x}_{n}=0, \forall n \notin \mathcal{N}$ with $\mathcal{N}$ being the subset of transmitting antennas with cardinality equal to the number of available RF chains $\operatorname{card}(\mathcal{N})=N_{R F}$.

\section{Constructive Interference Classification AND BEAMFORMING}

Classical signal processing approaches regard interference as a disrupting element for transmissions and aim to reduce or nullify its effects over downlink transmissions through precoding [45]-[48]. In opposition to this, we employ a transmission concept where interference is seen as a resource to exploit and efficiently capitalize on to enhance system performances. More specifically, CI is used as a means to significantly increase the received signal-tointerference and noise ratio (SINR) [28], hence allowing a more robust detection at the user side. The concepts of interference exploitation for PSK signals were first introduced for linear precoding [28] and further extended to M-MIMO TAS systems in [16]. Here, we first describe CI theoretical foundations in PSK modulations, and then present the proposed optimization problems that aim to exploit interference in a joint TAS-Precoded transmission. 


\section{A. CI for PSK signals}

For PSK-modulated signals, MUI can be deconstructed into the linear combination of two different elements: a constructive component, beneficial for the transmission, and destructive component, detrimental for reception. The distinction between the two components is performed according to simple geometrical concepts, which are described in details in CI literature [28], [29], [33].

The relationship between interference and desired constellation symbol can be explicitly expressed from the definition of received symbol in a noiseless scenario $r_{k}$ as follows

$$
r_{k}=\sum_{n=1}^{N} h_{n, k} x_{n}=u_{k}+t_{k},
$$

where $t_{k}$ identifies the interference suffered by the $k$ th user, as shown in Fig.2 for an 8-PSK symbol. With reference to Fig. 2, $r_{k}$ is considered to be benefiting from interference, i.e., to be affected by CI, when MUI causes the received symbol to be positioned further away from the decision thresholds of the desired constellation symbol $u_{k}$. On the other hand, $r_{k}$ is perturbed by destructive interference if its distance from the decision thresholds is shorter than the one of $u_{k}$. Equivalent analytical CI conditions to the visual presentation in Fig.2 can be defined for a generalized $M$-ordered PSK modulation as [29]

$$
\omega_{k} \triangleq \Re\left(t_{k} \cdot e^{-j \phi_{k}}\right) \tan \Phi-\left|\Im\left(t_{k} \cdot e^{-j \phi_{k}}\right)\right| \geq 0,
$$

where $\omega_{k}$ denotes the interference classification criterion for user $k^{4}$, the operators $\Re(\cdot)$ and $\Im(\cdot)$ identify the real and imaginary part of the complex argument, respectively, and $\Phi$ represents the central angle of the constellation sectors identified by the decision thresholds, which can be computed in function of the constellation order $M$ as $\Phi=\pi / M$. In (5), we can see that the received signal for the $k$-th user is phase-shifted according to the phase of the desired constellation symbol for the $k$-th user $u_{k}=$ $e^{j \phi_{k}}$. The phase-shift is a fundamental step, as it allows to isolate the effects of the interference between phase and amplitude, as shown in Fig. 2 where the notation $\tau=$ $t_{k} \circ u_{k}^{*}=\tau_{R}+j \cdot \tau_{I}$ is used to identify the phase shifted $k$-th interfering symbol with its real and imaginary parts.

The inequality (5) offers an analytical evaluation on how constructive or destructive the interfering symbol $t_{k}$ is for the $k$-th user. In fact, if $t_{k}$ leads to a negative $\omega_{k}$ in (5), it directly implies that the received symbol for the $k$-th user is destructively affected by the interference, causing $r_{k}$ to lie in the destructive region of the symbol constellation, the red area in Fig.2. On the other hand, if $\omega_{k}$ is positive (i.e., the inequality is respected), we can

\footnotetext{
${ }^{4}$ More specifically, $\omega_{k}$ is the $k$-th element of the $\mathbb{R}^{K \times 1}$ vector $\boldsymbol{\omega}=\Re\left(\mathbf{t} \circ \mathbf{u}^{*}\right) \tan \Phi-\left|\Im\left(\mathbf{t} \circ \mathbf{u}^{*}\right)\right|$, which collects the classification criterion for each of the $K$ users.
}

automatically infer that the received symbol is benefiting from CI, as MUI pushes $r_{k}$ further away from the decision thresholds (i.e., the blue region in Fig.2) and consequently more robust against noise effects. In other words, CI concepts allow the system to experience an increase in the received power, while keeping the transmitted power at the base station fixed. The reader is referred to [29] for a detailed description of the above geometry and the interference classification criteria.

\section{B. Constructive Interference Beamforming}

The concepts of MUI exploitation led to the design of a CI beamforming in [29], where the downlink beamforming problem was formulated in order to exploit the MUI experienced at the receiver side. More specifically, it was shown in [29] that CI concepts can be used in order to minimize the transmitted power required in order to achieve specific SINR requirements. Under these assumptions, the beamforming problem becomes

$$
\begin{array}{lll}
\mathcal{P}_{1}: & \underset{\mathbf{x}}{\operatorname{minimize}} & \|\mathbf{x}\|^{2} \\
& \text { subject to } & \left|\Im\left(\dot{\mathbf{h}}_{k}^{T} \mathbf{x}\right)\right| \leq\left[\Re\left(\dot{\mathbf{h}}_{k}^{T} \mathbf{x}\right)-\Gamma_{k}\right] \tan \Phi, \forall k,
\end{array}
$$

where $\dot{\mathbf{h}}_{k}=\mathbf{h}_{k} \cdot u_{k}^{*}$ and $\Gamma_{k}, \forall k$ identifies the SINR constraints considered for the system. In [29] it was shown that $\mathcal{P}_{1}$ is a second-order cone programming problem and can be efficiently solved by means of standard convex optimization tools.

\section{Proposed Joint MiP Constructive Antenna SElECTION AND PRECOdING (MIP-CASP)}

In this section, we introduce a novel transmission scheme where CI conditions in (5) are considered to define a novel optimization problem where TAS and downlink precoding are jointly performed. The newly defined optimization problem is designed so that the minimum achievable value of CI among all the users is maximized, while simultaneously optimizing two separate optimization variables, i.e., TAS vector a and the corresponding precoded vector $\tilde{\mathbf{x}}$. Analytically, we have

$$
\begin{aligned}
& \mathcal{P}_{2}: \underset{\mathbf{a}, \tilde{\mathbf{x}}}{\operatorname{maximize}} \min \left\{\Re\left(\mathbf{t} \circ \mathbf{u}^{*}\right) \tan \Phi-\left|\Im\left(\mathbf{t} \circ \mathbf{u}^{*}\right)\right|\right\} \\
& \text { subject to } \mathbf{t}=\mathbf{H}^{T} \tilde{\mathbf{x}}-\mathbf{u} \text {, } \\
& \|\tilde{\mathbf{x}}\|^{2} \leq 1 \\
& |\tilde{\mathbf{x}}| \preceq \mathbf{a} \\
& \sum_{n=1}^{N} a_{n}=N_{R F} \text {, } \\
& a_{n} \in\{0,1\},
\end{aligned}
$$

where the operator $\circ$ identifies the Hadamard product, $\mathbf{b} \preceq \mathbf{c}$ represents that inequality has to be respected for each element of both vectors (i.e., $b_{i} \leq c_{i}, \forall i$ ) and a represents the selection vector, whose entries are either 
one, when the corresponding antenna is to be connected to the RF chain for transmission, or null, when the corresponding antenna needs to be deactivated. As we can see, $\mathcal{P}_{2}$ maximizes the minimum value of the $\mathrm{CI}$ criterion for all the users $\boldsymbol{\omega}$. In fact, when the minimum value of $\boldsymbol{\omega}$ is positive, CI conditions in (5) are met and maximized for all the users. On the other hand, when the solution to $\mathcal{P}_{2}$ leads to negative values of the cost function, it implies that the achieved solution is minimizing destructive interference effects as its least constructive component is maximized. Clearly, (7) jointly optimizes the precoded symbols through $\tilde{\mathbf{x}}$ and TAS through a, subject to power constraints in (7c) (without loss of generality we assume a total power budget of 1 for simplicity), and the typical antenna-number constraint also found in (21). Additionally, the relationship between precoded vector and TAS vector is represented by the constraint (7d), which imposes the elements of $\tilde{\mathbf{x}}$ to be null when corresponding to a deselected antenna. Given its binary constraint, the optimization problem (7) is clearly nonconvex, however it can be efficiently solved by means of commercial optimization tools such as MoSek. Still, it is important to highlight that its objective function is concave w.r.t. $\tilde{\mathbf{x}}^{5}$ [29], since it can be deconstructed into the combination of two functions: a linear function $\Re\left(\mathbf{t} \circ \mathbf{u}^{*}\right)$ and a concave function $-\left|\Im\left(\mathbf{t} \circ \mathbf{u}^{*}\right)\right|$, as the extraction of the imaginary and real of a linear function preserves its linearity [29].

As we can see, $\mathcal{P}_{2}$ is designed in order to jointly perform TAS (i.e., identifying the subset of transmitting antennas a) and design the precoded signal $\tilde{\mathbf{x}}$. The joint optimization allows us to fully exploit the beneficial components of MUI, achieving significant transmission benefits and a particularly interesting trade-off between system complexity and performances.

\section{Heuristic Approaches to Joint Antenna SELECTION AND PRECODING}

While the joint MIP-CASP approach above effectively reduces the RF chains at the transmitter, the joint optimization of $\mathbf{a}$ and $\tilde{\mathbf{x}}$ involved introduces a significant computational burden. Accordingly, we propose three heuristic successive optimization approaches, based on the decomposition of $\mathcal{P}_{2}$ into three different optimization problems. In line with the literature on multiple variable non-convex optimization [19], $\mathcal{P}_{2}$ can be heuristically solved by optimizing in a successive manner three separate single-variable optimization problems. Given the analytical definition of $\mathcal{P}_{2}$, the optimization problem can be approached by recursively solving the same optimization problem when one of the two variables (i.e., a and $\tilde{\mathbf{x}}$ )

\footnotetext{
${ }^{5}$ Note that the optimization variables directly affect the cost function This can be explicitly expressed by substituting the constraint (7d) into the cost function.
}

is considered fixed. That is, $\mathcal{P}_{2}$ can be decomposed into the succession of three convex optimization problems, as follows:

- a full-system preliminary precoding, where the precoded signal $\mathrm{x}$ of the system with no TAS is derived via constructive beamforming $(\mathrm{CBF})$

- TAS, where the sub-set of transmitting antennas a is identified via CI antenna selection (CAS)

- subset precoding, where the transmitted signal for the chosen $N_{R F}$ transmitting antennas $\tilde{\mathbf{x}}$ is re-computed 6

Towards reducing the involved computational complexity, the solution to the succession of these problems can be achieved through different approaches with decreasing computational complexities, here introduced and discussed. More specifically, we introduce the three following approaches with reducing computational complexity:

- 3-step approach, namely CBF-CAS-CBF, involving $\mathrm{CBF}$ for the original precoding in the first step, CAS in TAS step, and CBF in the final precoding step, where each one of the three aforementioned optimization problems is solved through convex optimization tools

- 2-step approach, namely MFCAS-CBF, where the first step is circumvented by employing a closed form Matched Filtering (MF) precoder, while the remaining two problems are solved by means of convex optimization techniques

- 1-step approach, namely MFCAS, where the first and last steps are circumvented by employing the MF precoder and only the antenna optimization problem is solved by convex optimization.

It is important to highlight that all the proposed heuristic approaches converge. In fact, the proposed algorithms are based on the successive optimization of convex optimization problems, whose optimality and convergence are known, as shown in detail in [49].

\section{A. 3-step Successive Optimization CBF-CAS-CBF}

We define as 3-step CBF-CAS-CBF the scheme based on the decomposition of $\mathcal{P}_{2}$ into three different convex problems to be solved in a sequential manner. In 3-step CBF-CAS-CBF, we first derive the precoded vector for the full-system $\mathbf{x}=\left[x_{1}, \ldots, x_{N}\right]^{T}$ by solving the $\mathrm{CBF}$ optimization problem

$$
\begin{aligned}
\mathcal{P}_{3 a}: \quad \begin{array}{c}
\operatorname{maximize}_{\mathbf{x}} \\
\text { subject to }
\end{array} & \mathbf{m i n}\left\{\Re\left(\mathbf{t} \circ \mathbf{H}^{T} \mathbf{x}-\mathbf{u}\right) \tan \Phi-\left|\Im\left(\mathbf{t} \circ \mathbf{u}^{*}\right)\right|\right\} \\
& \|\mathbf{x}\|^{2} \leq 1 .
\end{aligned}
$$

${ }^{6}$ While TAS is intrinsically an integer programming problem, it can be approached by relaxing the integer constraints over the selection vector a, in line with [19]. In fact, if we relax the binary constraints over a into a continuous positive real value, the optimization problem becomes convex, allowing us to solve it via conventional convex optimization tools. 
The optimization problem $\mathcal{P}_{3 a}$ can be cast as a secondorder cone programming [49], since its objective function is concave [29]. Once the optimal precoded vector for the full $N$-antenna system $\mathrm{x}$ is achieved, the system proceeds in identifying the antenna subset for transmission based on a. The constructive TAS is performed according to the following CAS optimization problem

$$
\begin{aligned}
\mathcal{P}_{3 b}: \begin{array}{c}
\text { maximize } \\
\text { subject to }
\end{array} & \min \left\{\Re \left(\mathbf{t} \circ \mathbf{H}^{*} \mathbf{x}-\mathbf{u},\right.\right. \\
& |\mathbf{x}| \preceq \mathbf{a} \\
& a_{n} \in[0,1], \forall n \in\{1, \ldots, N\} \\
& \sum_{n=1}^{N} a_{n}=N_{R F},
\end{aligned}
$$

where a represents the selection vector, following the same notation as for $\mathcal{P}_{2}$. The solution to $\mathcal{P}_{3 b}$ yields a vector with non-binary values of $\mathbf{a}$, which are achieved by selecting the $N_{R F}$ largest elements with their indices representing the selected antennas. Finally, in order to achieve the final transmitted signal, the solution to $\mathcal{P}_{3 b}$ is used to identify the precoded vector $\tilde{\mathbf{x}}$ for the transmitting antennas subset $\mathcal{N}$ in the following

$$
\begin{aligned}
\mathcal{P}_{3 c}: \quad \underset{\tilde{\mathbf{x}}}{\operatorname{maximize}} & \min \left\{\Re\left(\mathbf{t} \circ \mathbf{u}^{*}\right) \tan \Phi-\left|\Im\left(\mathbf{t} \circ \mathbf{u}^{*}\right)\right|\right\} \\
\text { subject to } & \mathbf{t}=\mathbf{H}^{T} \tilde{\mathbf{x}}-\mathbf{u} \\
& \|\tilde{\mathbf{x}}\|^{2} \leq 1 \\
& |\tilde{\mathbf{x}}| \preceq \mathbf{a} .
\end{aligned}
$$

\section{B. 2-step Successive Optimization MFCAS-CBF}

While the previous approach is able to achieve near optimal performances, it is based on the derivation of the precoding vector for the full-size system $\mathbf{x}$, which is a computationally demanding step. Because of this, in order to further reduce the computational complexity of the signal processing at the BS, we propose an additional approach, called 2-step MFCAS-CBF, which leverages on the known property of asymptotic optimality for linear precoding in M-MIMO systems [2].

Thanks to this property, the computational burdens required by the convex precoding in $\mathcal{P}_{3 a}$ are greatly reduced, as they are replaced by a simple closed-form linear precoding approach. As it follows, we can represent the 2-step MFCAS-CBF approach in the following algorithm, where we first identify the subset of transmitting antennas based on the assumption of MF precoding

$$
\begin{aligned}
& \mathcal{P}_{4 a}: \underset{\Delta}{\operatorname{maximize}} \min \left\{\Re\left(\mathbf{c} \circ \mathbf{u}^{*}\right) \tan \Phi-\left|\Im\left(\mathbf{c} \circ \mathbf{u}^{*}\right)\right|\right\} \\
& \text { subject to } \mathbf{c}=\mathbf{H}^{T} \boldsymbol{\Delta} \mathbf{H u}-\mathbf{u} \text {, } \\
& \sum_{n=1}^{N} \Delta_{n, n}=N_{R F} \text {, } \\
& \Delta_{n, n} \in[0,1] \text {, }
\end{aligned}
$$

where $\Delta$ is a $N \times N$ selection matrix and $c_{k}$ is the $k$-th element of the MUI interference with MF assumption $\mathbf{c}=$
$\mathbf{H}^{T} \boldsymbol{\Delta} \mathbf{H u}-\mathbf{u}$, i.e., $\mathbf{x}=\mathbf{H u}$. Matrix $\boldsymbol{\Delta}$ is real-valued and diagonal, and its entries can be either null, i.e., $\Delta_{n, n}=0$ if $n$ is a non-selected antenna, or unitary, i.e., $\Delta_{n, n}=1$ if $n$ is an antenna selected for transmission. After identifying the antenna subset $\mathbf{a}=\operatorname{diag}(\boldsymbol{\Delta})$, we then proceed in deriving the precoding vector $\tilde{\mathbf{x}}$ as a solution to the $\mathrm{CBF}$ problem

$$
\begin{aligned}
\mathcal{P}_{4 b}: \underset{\tilde{\mathbf{x}}}{\operatorname{maximize}} & \min \left\{\Re\left(\mathbf{t} \circ \mathbf{u}^{*}\right) \tan \Phi-\left|\Im\left(\mathbf{t} \circ \mathbf{u}^{*}\right)\right|\right\} \\
\text { subject to } & \mathbf{t}=\mathbf{H}^{T} \tilde{\mathbf{x}}-\mathbf{u}, \\
& \|\tilde{\mathbf{x}}\|^{2} \leq 1 \\
& |\tilde{\mathbf{x}}| \preceq \mathbf{a}
\end{aligned}
$$

which can be efficiently solved by standard convex optimization techniques, as for in (10).

\section{1-step Successive Optimization MFCAS}

In addition to the previous schemes, we propose a final approach to MUI exploiting AS-precoding where the computational burden is further reduced. Here, the antenna subset selection is the only problem that requires convex optimization in order to be solved, while precoding is performed by assuming only $\mathrm{MF}$ at the transmitter side. Accordingly, we can define a new single-step optimization problem as follows

$$
\begin{aligned}
& \mathcal{P}_{5}: \underset{\boldsymbol{\Delta}}{\operatorname{maximize}} \min \left\{\Re\left(\mathbf{c} \circ \mathbf{u}^{*}\right) \tan \Phi-\left|\Im\left(\mathbf{c} \circ \mathbf{u}^{*}\right)\right|\right\} \\
& \text { subject to } \mathbf{c}=\mathbf{H}^{T} \boldsymbol{\Delta} \mathbf{H u}-\mathbf{u} \text {, } \\
& \sum_{n=1}^{N} \Delta_{n, n}=N_{R F} \\
& \Delta_{n, n} \in[0,1] \text {, }
\end{aligned}
$$

After the transmitting subset $\mathcal{N}$ has been identified, we proceed in computing the transmitted signal $\tilde{\mathbf{x}}$, based on $\mathrm{MF}$

$$
\tilde{x}_{n}=\left\{\begin{array}{lll}
1 / \xi_{n} \sum_{k=1}^{K} h_{n, k} u_{k}, & \forall & n \in \mathcal{N}, \\
0 & \forall & n \notin \mathcal{N},
\end{array}\right.
$$

where $\xi_{n}$ is a scaling factor, which guarantees a unitary transmitted power $\sum_{n=1}^{N}\left|x_{n}\right|^{2}=1$.

\section{Optimality EVALUATION}

In this section, we further characterize the proposed heuristic approaches by studying the impact that successive optimization and closed form approximations have over the achievable cost function values. In line with the literature [50], we define the figure of merit $\mathcal{M}$ as

$$
\mathcal{M}=\frac{f}{f_{M I P}^{\star},}
$$

where $f$ defines the objective function for which we want to measure the optimality. Clearly here

$$
f=\min \left\{\Re\left(\mathbf{t} \circ \mathbf{u}^{*}\right) \tan \Phi-\left|\Im\left(\mathbf{t} \circ \mathbf{u}^{*}\right)\right|\right\},
$$


represents the cost function of the optimization problem $\mathcal{P}_{2}$ when the heuristic solutions are considered, i.e., the minimum CI achieved by successive optimization techniques, and $f_{M I P}^{\star}$ identifies the cost function evaluation when the optimal MIP-CASP solution is considered, i.e., when $f$ is computed considering the $\tilde{\mathbf{x}}$ solution of $\mathcal{P}_{2}$. The defined metric represents a direct evaluation of the optimality of the proposed heuristic approaches, as $f_{M I P}^{\star}$ represents the optimal and maximum value of minimum CI achievable from a system. Clearly $\mathcal{M}=1$ signifies that a MIP-equivalent solution is obtained.

Fig.3 collects the cost function evaluation $f$ for all the proposed approached with several modulation orders when considering increasing sub-set array sizes at the base station, i.e., increasing the number of transmitting antennas at the BS $N_{R F}$. Interestingly, we can notice that both the 3-step and the 2-step approaches are able to achieve near optimal solutions when compared to the optimal $f_{M I P}^{\star}$ for all the modulation orders. This result is particularly important, as it proves that SO-based approximation approaches are able to efficiently approximate and solve the MIP equivalent formulation. On the other hand, we can see that the closed form single-step approach, 1-step MFCAS, is characterized by lower values of $f$ when we increase the modulation order, because of the suboptimal approach when solving the precoding problem (i.e., MF linear precoding). Nevertheless, Fig. 3 shows that such approach can still represent an interesting alternative for low-order modulation and low-energy scenarios, as it is characterized by very low complexity and is still able to achieve acceptable performances for the 4-PSK and 8PSK scenario.

These results are confirmed in Fig.4, where the figure of merit $\mathcal{M}$ curves are presented for three different modulation orders. As we can see, these results confirm that both the 2-step and 3-step approaches are characterized by near optimal performances, as they rapidly and closely approach the optimality line, represented by the unitary value. On the other hand, the 1-step MFCAS approach proves to be a valuable alternative for low-power and low-modulation scenarios, thanks to its favorable trade-off between complexity and performances. Towards quantifying this trade-off, below we evaluate the computational complexity of each of the proposed schemes.

\section{BENCHMARK SCHEMES}

In this section, we present the TAS-based benchmark schemes considered in the paper. In line with the literature [9], [14], they are all characterized by a cascade of stateof-the-art TAS algorithms and beamforming techniques.

Regarding TAS algorithms, we consider a near-optimal convex optimization-based capacity maximization [19] and a simple path gain amplitude maximization selection [11], [51]. TAS-based M-MIMO complexity could be further reduced by means of low-complex algorithms,

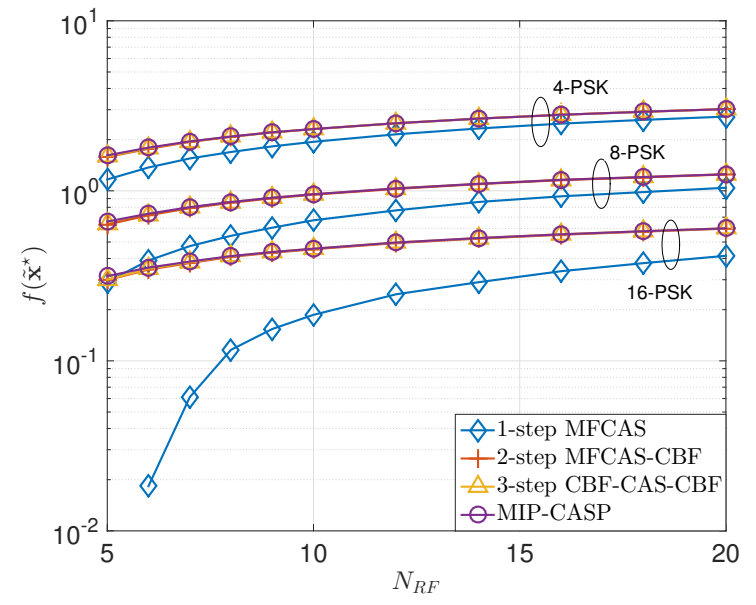

Fig. 3. Minimum CI when $K=5$ and $N=100$.

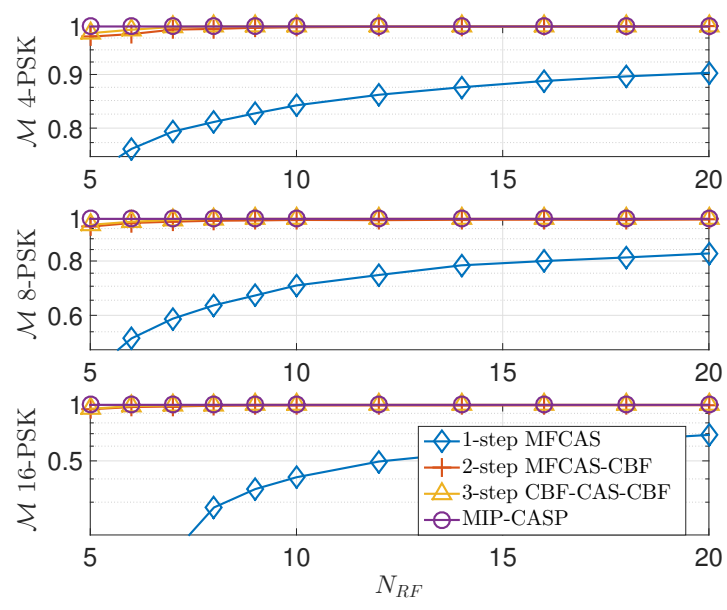

Fig. 4. Figure of merit $\mathcal{M}$ when $K=5$ and $N=100$.

such as the random selection described in [19] or TAS paired with matched filter precoding [16]. While these approaches identify interesting performances in terms of computational burdens [16], they are characterized by poor performance which severely affect the applicability in realistic scenarios.

Regarding beamforming techniques, two separate stateof-the-art algorithms are considered, both based on the optimization of the received SINR: transmitted power minimization [48] and SINR balancing [47]. More specifically, we can evaluate the received SINR for the $k$-th user as

$$
\gamma_{k}=\frac{\left|\mathbf{h}_{k}^{T} \mathbf{p}_{k}\right|^{2}}{\sum_{j \neq k}\left|\mathbf{h}_{k}^{T} \mathbf{p}_{j}\right|^{2}+N_{0}}=\frac{\left|\sum_{n=1}^{N} h_{n, k} p_{n, k}\right|^{2}}{\sum_{j \neq k}\left|\sum_{n=1}^{N} h_{n, k} p_{n, j}\right|^{2}+N_{0}}
$$

where $p_{n, k}$ represents the $n$-th element of the $k$-th user beamforming vector $\mathbf{p}_{k}$. Given the beamforming vector $\mathbf{p}_{k}$, it is possible to compute the transmitted signal $\mathbf{x}$ as 
follows

$$
\mathbf{x}=\sum_{k=1}^{K} \mathbf{p}_{k} u_{k} .
$$

\section{A. TAS: Capacity-Based Selection (CapMax)}

In [19], a convex approach to sum-capacity based antenna selection for M-MIMO is proposed. The selection is performed over the system sum-capacity when considering dirty-paper coding at the transmitter with equally distributed power among the users ${ }^{7}$. In particular, we can define the sum-capacity as

$$
\mathcal{E}=\log _{2}\left[\operatorname{det}\left(\mathbf{I}_{K}+\rho \mathbf{H}^{H} \boldsymbol{\Delta} \mathbf{H}\right)\right],
$$

where $\mathbf{I}_{K}$ is a $K$-dimensional identity matrix and $\boldsymbol{\Delta}$ is the selection diagonal matrix, as in $\mathcal{P}_{4 a}$. Accordingly, we can identify the optimization problem for sum-capacity maximization antenna selection as

$$
\begin{array}{rll}
\mathcal{P}_{6}: & \underset{\Delta}{\operatorname{maximize}} & \log _{2}\left[\operatorname{det}\left(\mathbf{I}_{K}+\rho \mathbf{H}^{H} \boldsymbol{\Delta} \mathbf{H}\right)\right] \\
& \text { subject to } & \Delta_{n, n} \in\{0,1\}, \\
& \sum_{n=1}^{N} \Delta_{n, n}=N_{R F} .
\end{array}
$$

Given its formulation, the optimization problem $\mathcal{P}_{6}$ is non-convex due to the binary constrains imposed over the diagonal of the selection matrix. Nevertheless, a relaxation of such constraint was proven to be achieving near-optimal performances when compared to exhaustive search approaches [19]. Accordingly, the new relaxed optimization problem can be defined as

$$
\begin{aligned}
& \mathcal{P}_{6}^{\prime}: \underset{\boldsymbol{\Delta}}{\operatorname{maximize}} \log _{2}\left[\operatorname{det}\left(\mathbf{I}_{K}+\rho \mathbf{H}^{H} \boldsymbol{\Delta} \mathbf{H}\right)\right] \\
& \text { subject to } \Delta_{n, n} \in[0,1] \text {, } \\
& \sum_{n=1}^{N} \Delta_{n, n}=N_{R F},
\end{aligned}
$$

which leads to TAS subset definition

$$
\mathcal{N}=\arg \max _{N_{t}}\left\{\Delta_{1,1}, \ldots, \Delta_{n, n}, \ldots, \Delta_{N, N}\right\} .
$$

The newly formulated optimization problem $\mathcal{P}_{6}^{\prime}$ is convex and can be solved by means of convex optimization tools [19]. A similar approach could be directly applied to the MIP-CASP problem. However, while such approach is characterized by an interesting low-complexity, we have chosen not to deviate from the focus of our paper, and designate this as the focus of our future work.

\section{B. TAS: Path Gain Selection (PGMax)}

Path gain selection at the transmitter can be easily performed by selecting the subset of antennas whose path gains are higher. The antenna subset can be analytically identified as follows

$$
\mathcal{N}=\arg \max _{N_{t}}\left\{\left\|\mathbf{h}_{1,:}\right\|^{2}, \ldots,\left\|\mathbf{h}_{n,:}\right\|^{2}, \ldots,\left\|\mathbf{h}_{N,:}\right\|^{2}\right\},
$$

\footnotetext{
${ }^{7}$ In the proposed benchmark schemes, power allocation is still performed at a latter stage, during beamforming design [47], [48].
}

where the notation $\mathbf{x}_{n,:}$ identifies the $n$-th row of the matrix $\mathbf{X}$ and $\max _{N_{R F}}$ identifies the $N_{R F}$ highest values of the argument.

\section{Beamforming: Transmitted Power Minimization Beam- forming}

Transmitted power minimization [48] represents a conventional approach to downlink beamforming, where interference is regarded to as a harmful element for transmission. Because of this, the beamforming optimization problem is designed to minimize the transmitted power while respecting predefined SINR requirements $\Gamma_{k}, \forall k \in$ $\{1, \ldots, K\}$. Analytically

$$
\begin{array}{ll}
\mathcal{P}_{7}: & \underset{\mathbf{p}_{k}}{\operatorname{minimize}} \\
& \sum_{k=1}^{K}\left\|\mathbf{p}_{k}\right\|^{2} \\
& \text { subject to } \quad \gamma_{k}=\frac{\left|\sum_{n=1}^{N} h_{n, k} p_{n, k}\right|^{2}}{\sum_{j \neq k}\left|\sum_{n=1}^{N} h_{n, k} p_{n, j}\right|^{2}+N_{0}} \geq \Gamma_{k},
\end{array}
$$

which can be efficiently solved by means of convex optimization with a semidefinite relaxation approach [48].

\section{Beamforming: SINR Balancing}

We define as SINR balancing the optimization problem that aims to maximize the minimum received SINR, while respecting a predefined transmitted power constraint $P_{T}$. The new problem can be analytically defined as

$$
\begin{aligned}
& \mathcal{P}_{8}: \underset{\mathbf{p}_{k}}{\operatorname{maximize}} \Gamma_{k} \\
& \text { subject to } \gamma_{k} \geq \Gamma_{k} \\
& \sum_{k=1}^{K}\left\|\mathbf{p}_{k}\right\|^{2} \leq P_{T} .
\end{aligned}
$$

Differently from $P_{7}$, the SINR balancing problem is non-convex and its solution requires a more complex algorithmic approach [47].

\section{ViII. Computational Evaluation}

We evaluate the computational costs of the proposed schemes in terms of running time for different antenna array sizes at the transmitter side. In order to perform a fair evaluation, we consider the running times in $s$ within a coherence time, i.e., for the number of frames where we can consider constant CSI. This is due to the fact that the proposed schemes require a symbol-rate evaluation of TAS/precoding, while the conventional CapMax scheme needs to be performed on a coherence time basis. With this regard, we consider a TDD scenario [52] and define as coherence time $T_{\text {cohe }}$ the total number of datasymbols that can be transmitted when channel propagation elements in $\mathbf{H}$ are constant. The assumption of a TDD scenario is common for M-MIMO, as frequency division duplexing would require more time for CSI acquisition 


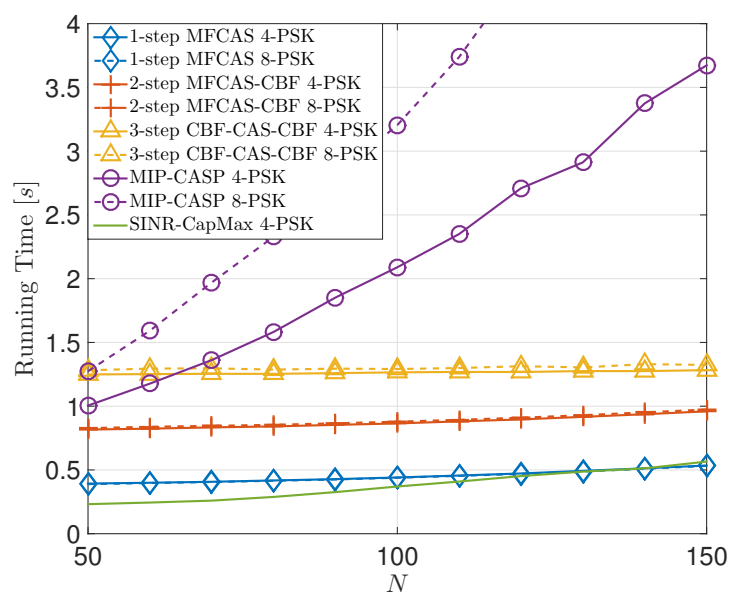

Fig. 5. Frame Running time when $K=5, N_{R F}=5$ and $T_{D L}=4$.

at the transmitter, hence reducing the number of symbols $T_{\text {data }} \ll T_{\text {cohe }}$ dedicated to data transmission [52]. On the other hand, the TDD assumption allows us to exploit the reciprocity of the channel, hence defining the number of slots used for CSI acquisition $T_{C S I}$ with a direct proportionality to the number of users $K$, instead of the numbers of antennas at the transmitter $N$. Accordingly, we have

$$
T_{\text {data }}=T_{\text {cohe }}-\mu K,
$$

where $\mu \geq 1$ is a parameter that defines the number of pilot symbols used for CSI per user. However, only a fraction $\eta_{D L}$ of $T_{\text {data }}$ is actually used in downlink transmission, thus defining the data symbols for downlink within a single coherence time as

$$
T_{D L}=\eta_{D L}\left(T_{\text {cohe }}-T_{C S I}\right) .
$$

When evaluating the coherence time dedicated to downlink transmission $T_{D L}$, we refer to realistic values for a fast-fading scenario (i.e., where the proposed schemes are mostly suited for) where $T_{\text {cohe }}=10$ symbols, in line with the work in [52]. More specifically, we consider a CSI acquisition time of $T_{C S I}=5$ symbols (i.e., $\mu=1$ ) in a DL dominant scenario with $T_{D L}=4$ symbols dedicated to downlink transmission (i.e., $\eta_{D L}=0.8$, with $T_{U L}=1$ ).

As we can see in Fig.5, the proposed schemes are overall affected by longer computational times over the length of the coherence time. This is due to the fact that the proposed schemes require a symbol-by-symbol update, in contrast with conventional TAS schemes from the literature. Nevertheless, it is interesting to notice that the proposed 1-step MFCAS scheme is characterized by running times that can be compared to the ones of the benchmark scheme. This strongly reaffirms that such approach represents a particularly appealing scheme for low-modulation scenarios, as it is able to achieve interesting performances with non-significant additional computational costs. On the other hand, it is important to notice how the proposed 2-step and 3-step schemes, are almost unaffected by the increase in array sizes, while the SINR-CapMax is instead characterized by increasing computational times as $N$ grows. Accordingly, for very large systems, the proposed schemes are expected to be characterized by similar complexity, when compared to existing TAS schemes. On the other hand, we can see that the MIP-CASP approach is characterized by higher computational times, because of its trellis search-based solution.

Remark. SINR-CapMax requires further operations at the receiver side in order to equalize the received signal. Because of this, BS needs to feed-forward the $k$-th user with the product of the channel with the $k$-th precoding vector, i.e., $\mathbf{h}_{k}^{T} \mathbf{p}_{k}, \forall k$, in order to recover the data. For CI precoding there is no need to equalize the composite channel, as the $r_{k}$ resides in the constructive area of the constellation. Accordingly, such feedback is not required by the CI approaches, where all complexity resides at the BS, which also makes them robust to the estimation and quantization errors that are involved in the feedforwarding process for conventional beamformers.

\section{Channel UnCERTAinty And Robust APPROACH}

In this section, we study the effects of imperfect CSI acquisition at the transmitter. More specifically, we define the channel uncertainty model and derive a robust precoding technique to counteract the errors caused by imperfect CSI.

\section{A. Model and Effects}

In our studies, imperfect CSI at the transmitter side is modelled by adding a complex random component to the channel matrix $\mathbf{H}$. Without loss of generality, we consider the case where channel uncertainty amplitude is upper bounded by a specific value, i.e., CSI at the BS is affected by spherical noise [29].

Accordingly, the estimated channel gain between the $n$-th antenna and the $k$-th user is analytically defined as

$\hat{h}_{n, k}=h_{n, k}+e_{n, k}, \forall n \in\{1, \ldots, N\}, \forall k \in\{1, \ldots, K\}$,

where $\hat{h}_{n, k}$ represents the channel gain estimation available at the BS and $e_{n, k}$ represents the channel uncertainty, i.e., $\sum_{n=1}^{N}\left|e_{n, k}\right|^{2} \leq \delta_{k}^{2}$ with $\delta_{k}$ being the uncertainty upper bound over the channel estimation for the $k$-th user.

Clearly, the presence of uncertainty over the available CSI at the BS has negative effects over the performances of a system. In fact, if we consider the BS to possess imperfect CSI as modelled, the received signal in a noise free scenario becomes

$$
\hat{r}_{k}=\sum_{n=1}^{N} \hat{h}_{n, k} x_{n}=\sum_{n=1}^{N} h_{n, k} x_{n}+\sum_{n=1}^{N} e_{n, k} x_{n},
$$


where the second term of the last equation explicitly represents effects of imperfect CSI at the transmitter side during signal processing.

In line with the literature [29], [53], BS is assumed to have no knowledge over the channel uncertainty $e_{n, k}, \forall n \in\{1, \ldots, N\}, k \in\{1, \ldots, K\}$ beside the upper bound $\delta_{k}$ for $k$-th channel. This assumption allows to derive a robust precoding design, which guarantees the downlink transmission to be resistant against all possible channel uncertainties within the upper bound $\delta_{k}^{2}$.

In the following, we propose a CSI-robust TASprecoding technique where we aim to minimize the transmitted power $P_{t}$ required to meet the constraints imposed by the specific optimization.

\section{B. MIP-CASP Robust Scheme}

Given the MIP-based optimization in (7), we can identify a worst-case design for imperfect CSI scenarios as

$$
\begin{aligned}
\mathcal{P}_{9}: \begin{array}{c}
\text { minimize } \\
\text { abject to }
\end{array} & \|\tilde{\mathbf{x}}\|^{2} \\
& \min _{\left\|\mathbf{e}_{k}\right\|^{2} \leq \delta_{,}^{2}, \forall k}\left\{\Re\left(\hat{\mathbf{t}}^{\prime}\right) \tan \Phi-\left|\Im\left(\hat{\mathbf{t}}^{\prime}\right)\right|\right\} \succeq 0, \\
& \hat{\mathbf{t}}^{\prime}=\left(\hat{\mathbf{H}}^{T} \tilde{\mathbf{x}}-\mathbf{u}\right) \circ \mathbf{u}^{*}, \\
& \|\tilde{\mathbf{x}}\|^{2} \leq 1, \\
& |\tilde{\mathbf{x}}| \preceq \mathbf{a}, \\
& \sum_{n=1}^{N} a_{n}=N_{R F}, \\
& a_{n} \in\{0,1\},
\end{aligned}
$$

where the super-index $\mathbf{b}^{\prime}=\mathbf{b} \circ \mathbf{u}^{*}$ is used to represent that the vector $\mathbf{b}$ has been phase shifted according to the desired symbols $\mathbf{u}$.

Because of the infinite number of possible error values $e_{n, k}$, the first constraint in $\mathcal{P}_{9}$ is intractable. However, by employing a worst-case approach, it is possible to derive a MIP-CASP robust design optimization for TAS-precoding with CI exploitation. In order to do so, we need to identify the equivalent constraint for a worst-case scenario, where the largest error is considered.

Considering an imperfect CSI and noiseless scenario, received symbols can be decomposed in order to explicitly show their real and imaginary part as follows

$$
\begin{aligned}
\hat{y}_{k} & =\sum_{n=1}^{N} \hat{h}_{k, n} x_{n} \\
& =\sum_{n=1}^{N}\left(\hat{h}_{k, n}^{R} x_{n}^{R}-\hat{h}_{k, n}^{I} x_{n}^{I}\right)+j\left(\hat{h}_{k, n}^{R} x_{n}^{I}+\hat{h}_{k, n}^{I} x_{n}^{R}\right),
\end{aligned}
$$

where real and imaginary part can be rearranged in order to explicitly identify the effects of imperfect CSI over the received symbols as

$$
\Im\left\{\hat{y}_{k}\right\}=\sum_{n=1}^{N}\left(h_{k, n}^{R} x_{n}^{R}-h_{k, n}^{I} x_{n}^{I}\right)+\left(e_{k, n}^{R} x_{n}^{R}-e_{k, n}^{I} x_{n}^{I}\right)
$$

$$
\Re\left\{\hat{y}_{k}\right\}=\sum_{n=1}^{N}\left(h_{k, n}^{R} x_{n}^{I}+h_{k, n}^{I} x_{n}^{R}\right)+\left(e_{k, n}^{R} x_{n}^{I}+e_{k, n}^{I} x_{n}^{R}\right) .
$$

Both (32) and (33) can be presented in a more compact manner by exploiting auxiliary vectors, which lead to the following set of equations

$$
\begin{gathered}
\Im\left\{\hat{y}_{k}\right\}=\hat{\mathbf{f}}_{k}^{T} \mathbf{w}_{1}=\mathbf{f}_{k}^{T} \mathbf{w}_{1}+\overline{\mathbf{e}}_{k}^{T} \mathbf{w}_{1} \\
\Re\left\{\hat{y}_{k}\right\}=\hat{\mathbf{f}}_{k}^{T} \mathbf{w}_{2}=\mathbf{f}_{k}^{T} \mathbf{w}_{2}+\overline{\mathbf{e}}_{k}^{T} \mathbf{w}_{2},
\end{gathered}
$$

where the vectors $\mathbf{f}_{k}=\left[\mathbf{h}_{k}^{R}, \mathbf{h}_{k}^{I}\right]^{T}$ and $\overline{\mathbf{e}_{k}}=\left[\mathbf{e}_{k}^{R}, \mathbf{e}_{k}^{I}\right]^{T}$ represent the real-valued $k$-th column of the channel matrix and the corresponding channel estimation error vector respectively, with $\hat{\mathbf{f}}_{k}=\mathbf{f}+\overline{\mathbf{e}_{k}}$. In a similar manner, $\mathbf{w}_{1}=\left[\mathbf{x}^{I}, \mathbf{x}^{R}\right]^{T}$ and $\mathbf{w}_{2}=\left[\mathbf{x}^{R},-\mathbf{x}^{I}\right]^{T}$ are two auxiliary real-valued representations of the precoded vector.

Likewise, we can rewrite the first constraint of $\mathcal{P}_{9}$ with the same notation

$$
\begin{array}{r}
\Im\left(\hat{t}_{k} e^{-j \phi_{k}}\right)=\dot{\hat{\mathbf{f}}}^{T} \mathbf{w}_{1}=\dot{\mathbf{f}}_{k}^{T} \mathbf{w}_{1}+\dot{\mathbf{e}}^{T} \mathbf{w}_{1} \\
\Re\left(\hat{t}_{k} e^{-j \phi_{k}}\right)=\dot{\hat{\mathbf{f}}}^{T} \mathbf{w}_{2}=\dot{\mathbf{f}}_{k}^{T} \mathbf{w}_{2}+\dot{\overline{\mathbf{e}}}^{T} \mathbf{w}_{2}-1,
\end{array}
$$

where

$$
\begin{aligned}
\dot{\mathbf{f}}_{k} & =\left[\left(\mathbf{h}_{k}^{I} u_{k}^{R}-\mathbf{h}_{k}^{R} u_{k}^{I}\right),\left(\mathbf{h}_{k}^{R} u_{k}^{R}+\mathbf{h}_{k}^{I} u_{k}^{I}\right)\right]^{T} \\
\dot{\overline{\mathbf{e}}}_{k} & =\left[\left(\overline{\mathbf{e}}_{k}^{I} u_{k}^{R}-\overline{\mathbf{e}}_{k}^{R} u_{k}^{I}\right),\left(\overline{\mathbf{e}}_{k}^{R} u_{k}^{R}+\overline{\mathbf{e}}_{k}^{I} u_{k}^{I}\right)\right]^{T}
\end{aligned}
$$

represent the real-valued channel vector and CSI error vector for the $k$-th user, whose representations have been modified in order to include the phase shift. Without loss of generality, for the sake of simplicity of notation, from now on we consider $\dot{\overline{\mathbf{e}}}_{k}=\overline{\mathbf{e}}_{k}$. Accordingly, we can rewrite the first constraint of $\mathcal{P}_{9}$, as

$$
\min _{\left\|\mathbf{e}_{k}\right\|^{2} \leq \delta_{k}^{2}}\left\{\left(\dot{\mathbf{f}}_{k}^{T} \mathbf{w}_{2}+\overline{\mathbf{e}}_{k}^{T} \mathbf{w}_{2}-1\right) \tan \Phi-\left|\dot{\mathbf{f}}_{k}^{T} \mathbf{w}_{1}+\overline{\mathbf{e}}_{k}^{T} \mathbf{w}_{1}\right|\right\} \geq 0 .
$$

which can be equivalently decomposed into two different constraints

$$
\begin{aligned}
& \min _{\left\|\mathbf{e}_{k}\right\|^{2} \leq \delta_{k}^{2}}\left\{\left(\dot{\mathbf{f}}_{k}^{T} \mathbf{w}_{2}+\overline{\mathbf{e}}_{k}^{T} \mathbf{w}_{2}-1\right) \tan \Phi-\left(\dot{\mathbf{f}}_{k}^{T} \mathbf{w}_{1}+\overline{\mathbf{e}}_{k}^{T} \mathbf{w}_{1}\right)\right\} \geq 0 \\
& \min _{\left\|\mathbf{e}_{k}\right\|^{2} \leq \delta_{k}^{2}}\left\{\left(\dot{\mathbf{f}}_{k}^{T} \mathbf{w}_{2}+\overline{\mathbf{e}}_{k}^{T} \mathbf{w}_{2}-1\right) \tan \Phi+\left(\dot{\mathbf{f}}_{k}^{T} \mathbf{w}_{1}+\overline{\mathbf{e}}_{k}^{T} \mathbf{w}_{1}\right)\right\} \geq 0 .
\end{aligned}
$$

The assumption of a spherical error over the CSI acquisition allows us to derive a robust formulation for (41) and (42). In fact, the worst-case scenario is characterized by the channel errors to be $\left\|\mathbf{e}_{k}\right\|^{2}=\delta_{k}^{2}, \forall k$, hence causing the constraints to be lower-bounded by the following equations

$$
\begin{aligned}
& {\left[\left(\dot{\mathbf{f}}_{k}^{T} \mathbf{w}_{2}-1\right) \tan \Phi-\dot{\mathbf{f}}_{k}^{T} \mathbf{w}_{1}\right]-\delta\left\|\mathbf{w}_{1}-\mathbf{w}_{2} \tan \Phi\right\| \underset{(43)}{\geq 0}} \\
& \left.\left[\left(\dot{\mathbf{f}}_{k}^{T} \mathbf{w}_{2}-1\right) \tan \Phi+\dot{\mathbf{f}}_{k}^{T} \mathbf{w}_{1}\right]-\delta \| \mathbf{w}_{1}+\mathbf{w}_{2} \tan \Phi\right) \| \underset{(44)}{\geq 0 .}
\end{aligned}
$$

Thanks to the new robust formulation for the constraints 
of $\mathcal{P}_{9}$, we can derive a MIP representation of the worstcase design for imperfect CSI scenarios that can be efficiently solved by means of optimization tools as its nonrobust counterpart. More specifically, the optimization problem $\mathcal{P}_{9}$ in its MIP representation becomes

$$
\begin{aligned}
& \mathcal{P}_{9}^{*}: \underset{\mathbf{a}, \mathbf{w}_{1}, \mathbf{w}_{2}}{\operatorname{minimize}} \sum_{n}^{N_{R F}}\left\|\mathbf{w}_{1}\right\|^{2} \\
& \text { subject to Constraints (43) and (44) }
\end{aligned}
$$

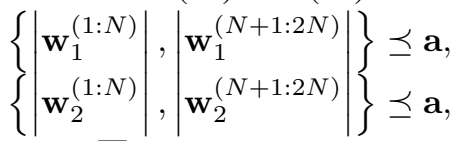

$$
\begin{aligned}
& \mathbf{w}_{1}=\Pi \mathbf{w}_{2} \text {, } \\
& \sum_{n=1}^{N} a_{n}=N_{R F}, \quad a_{n} \in\{0,1\},
\end{aligned}
$$

where $\boldsymbol{\Pi}=\left[\mathbf{0}_{N},-\mathbf{I}_{N} ; \mathbf{I}_{N}, \mathbf{0}_{N}\right], \mathbf{a}^{(1: N)}$ notation is used to identify the new vector $\mathbf{b}=\left[a_{1}, \ldots, a_{N}\right]$ and $\{\mathbf{a}, \mathbf{b}\} \preceq \mathbf{c}$ is used to impose the inequality to both vectors (i.e., $\mathbf{a} \preceq \mathbf{c}$ and $\mathbf{b} \preceq \mathbf{c}$ ).

\section{Results}

In this section the performances of the proposed transmission schemes are presented and discussed. The shown results are evaluated through Monte Carlo simulations over 50000 channel realizations. In order to study the performances of the proposed schemes, we evaluate the SER at the receiver side, the achievable capacity and the power efficiency of the system. More specifically, we present results for both 4-PSK and 8-PSK, as the proposed transmission schemes can be directly applied to any PSK modulation order. Legends are characterized by the following notation:

- MIP-CASP identifies the CI exploitation transmission scheme based on MIP,

- 3-step CBF-CAS-CBF is used to represent the CI transmission based on the solution of $\mathcal{P}_{3}$,

- 2-step MFCAS-CBF represents the 2-step TASprecoding heuristic scheme,

- 1-step MFCAS is used to classify the single-step approach

- SINR-CapMax stands for the literature approach where TAS is performed by CapMax and precoding is performed through SINR balancing.

Moreover, we compare the proposed schemes with two low-complexity additional approaches from the literature: ZF-PGMax, where zero forcing (ZF) linear precoding is considered and TAS is performed via path gain selection (PGMax), and HY-CIM from [16] for the 4-PSK scenarios, where hybrid linear precoding is considered (HY) and TAS is performed in order to maximize constructive interference (CIM). In our simulations, we consider a single-cell downlink M-MIMO scenario, where the BS possess perfect CSI and employs a VLA of $N=100$ antennas and communicates with $K=5$ single-antenna mobile users, unless differently specified.

\section{A. Symbol Error Rate}

In Fig. 6 we compare the SER of the proposed and conventional approaches for the case of 4-PSK modulation. The proposed schemes greatly outperform all the benchmark techniques, including SINR-CapMax, which is characterized by a combination of CapMax TAS [19] and SINR-balancing beamforming at the transmitter side [47]. At the same time, both 2-step MFCAS-CBF and 3-step CBF-CAS-CBF are able to achieve near optimal performances when compared to the MIP-CASP approach. This is supported by the previous results in terms of $\mathcal{M}$, which showed how the two heuristic approaches were able to achieve similar performances to the MIP-based scheme. On the other hand, 1-step MFCAS obtains reasonable performance in the relatively low-to-mid SNR range, as the error-floor of the MF is reached when SER is lower than $10^{-4}$. This is due to 4-PSK wider CI regions, which allow a relative robustness against the inability of MF precoding to efficiently separate the stream between the users. However, such inability becomes the main cause for errors at high SNR and leads to the typical error-floor. This confirms our previous considerations regarding 1-step MFCAS as a valuable approach for the low-complexity and low-power scenarios.

In Fig.7, we explore the same set-up for 8-PSK modulation. The performance trends for the proposed techniques are preserved. In fact, all the schemes based on CI exploitation are able to outperform both the SINRCapMax and the ZF-PGMax schemes. At the same time, the error floor for the 1-step MFCAS approach is higher than the one achieved in the 4-PSK case. This is due to the fact the final closed-form MF precoding is not able to correctly separate the different data-streams for different users, hence leading to uncontrolled inter-channel interference, whose effects are more visible in higher modulation orders. It is important to highlight that tighter decision thresholds also affect the performance gap between MIPCASP and heuristic approaches. In fact, even though heuristic approaches are characterized by high figures of merit, as shown in Fig.4, their impact over the SER performances differ according to the modulation order. In fact, while the figures of merit for 4-PSK and 8-PSK are comparable, the SER performance gap is almost negligible in the 4-PSK case in Fig.6, becoming more pronounced for the 8-PSK case in Fig.7.

\section{B. Data Rate}

As the conventional CapMax approach is designed for capacity maximization, it is important to compare the rate performance of the proposed and conventional schemes. Accordingly, we compare the throughput of the 


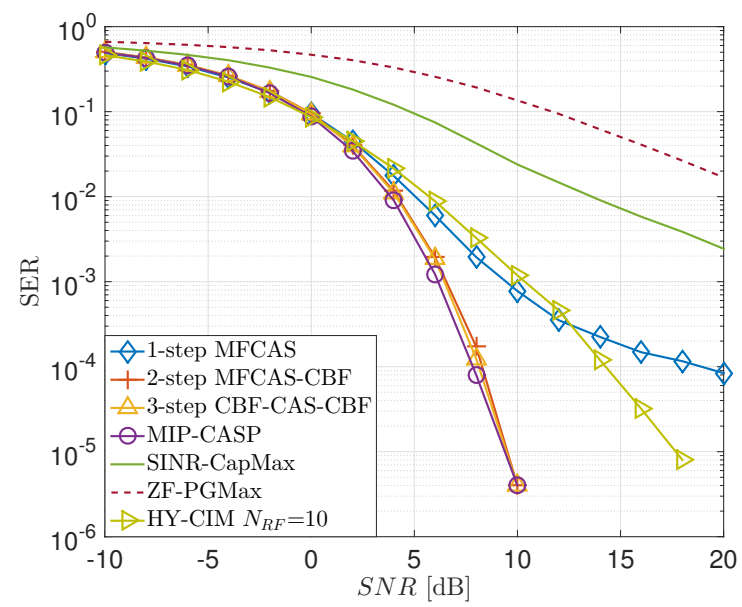

Fig. 6. 4-PSK Symbol Error Rate when $K=5, N=100$ and $N_{R F}=$ 5 with perfect CSI.

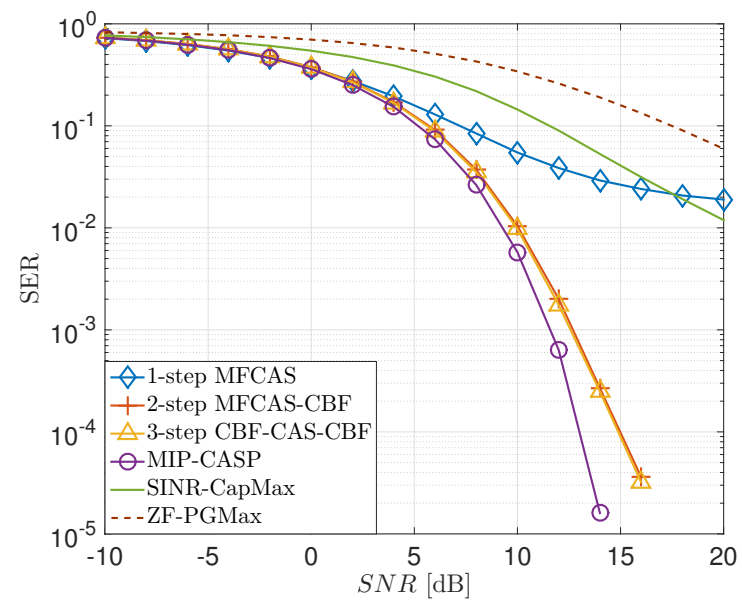

Fig. 7. 8-PSK Symbol Error Rate when $K=5, N=100$ and $N_{R F}=$ 5 with perfect CSI.

MIP-CASP scheme with the capacity achievable when considering the CapMax TAS scheme from the literature. The use of throughput instead of the ergodic capacity, i.e., $\sum_{k} \log _{2}\left(1+\gamma_{k}\right)$, as a performance metric for the proposed MIP-CASP is justified by the fact that its assumption of a specific modulation, i.e., any PSK modulation order, does not allow to support the assumption of Gaussian signals. We define the throughput as [28]

$$
T=(1-B L E R) \cdot m \cdot K,
$$

where $B L E R$ is the block error rate, $m=\log _{2}(M)$ is the bit information per symbol and $K$ is the number of users in the chosen scenario.

Performances are presented in Fig.8, where we compare the throughput of the proposed MIP-CASP approach for increasing modulation order, with the capacity of the full system and that of the CapMax selection. The solid line with circular markers in the figure represents the peakthroughput trend for the proposed approach, showing that the proposed scheme with increasing modulation orders outperforms the CapMax selection from the literature. It

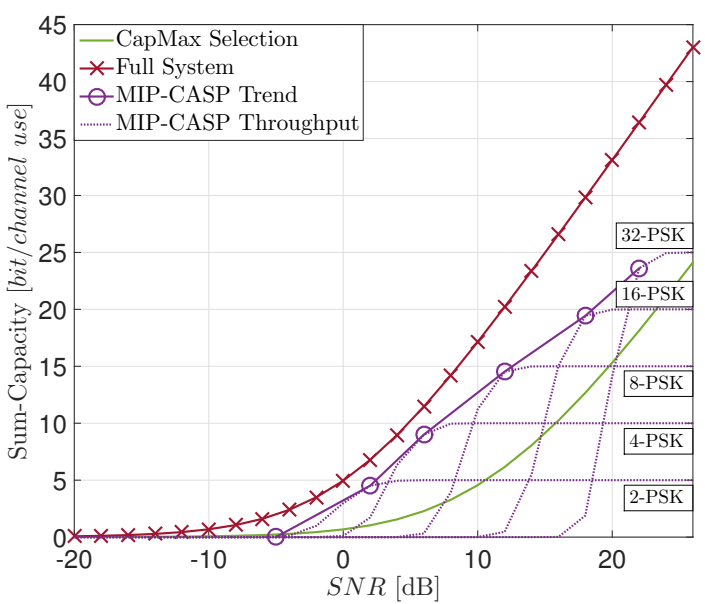

Fig. 8. System Capacity comparison when $K=5, N_{R F}=5$ and $N=100$.

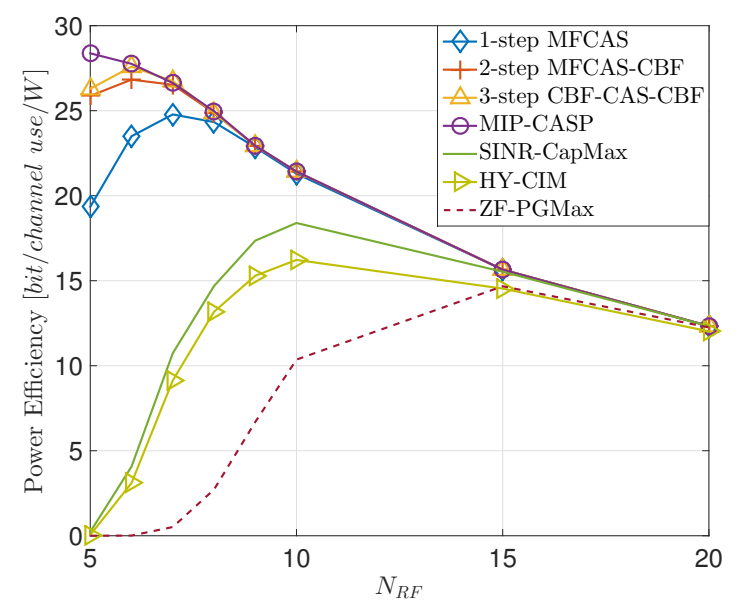

Fig. 9. 4-PSK Power Efficiency $\eta_{T}$ when $K=5, N=100$ with perfect CSI and $S N R=5 d B$.

is important to notice that the proposed scheme is able to achieve performances that are comparable to the ones of a full-system for low-to-mid SNR scenarios, where the gap with the CapMax scheme from the literature is more pronounced.

\section{Power Efficiency}

In order to better highlight benefits and trade-offs brought by the proposed schemes, we introduce a parameter which combines the achieved throughput with the power consumption at the transmitter side. More specifically, we employ a simple power efficiency metric as $\eta_{T}$

$$
\eta_{T}=\frac{T}{P_{a m p}+N_{R F} \cdot P_{R F}},
$$

where $P_{a m p}[W]$ is the power consumption of the amplifier, $P_{R F}[W]$ is the power requirement of one of the RF chains employed at the transmitter side, which is characterized by digital-analog converter, mixer and filter. When computing (47), we consider realistic power 


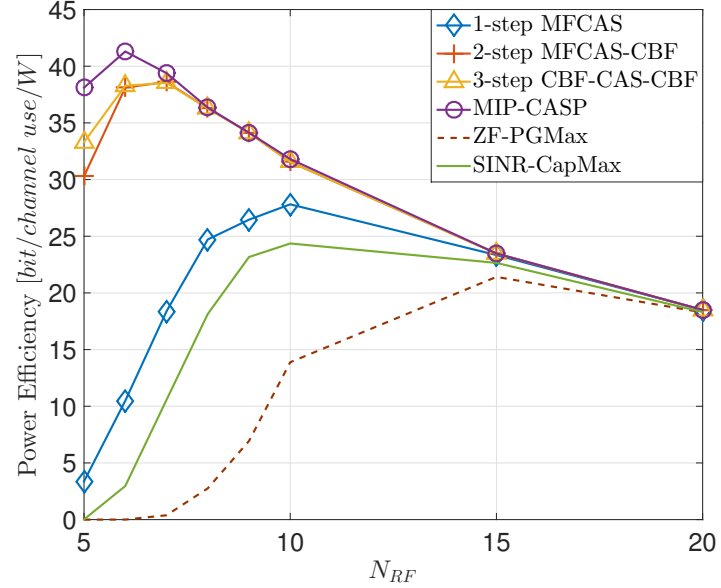

Fig. 10. 8-PSK Power Efficiency $\eta_{T}$ when $K=5, N=100$ with perfect CSI and $S N R=10 \mathrm{~dB}$.

values from practical systems [54], where $P_{a m p}=P_{t} / \nu$ is defined as the power required by an amplifier with $\nu=0.35$ efficiency and transmitted power $P_{t}=30 \mathrm{dBm}$ and $P_{R F}=65.9 \mathrm{~mW}$. Performances are presented in Fig.9 and Fig.10 as a function of $N_{R F}$ with $S N R=5 d B$ and $S N R=10 d B$, respectively. The proposed metric allows to better characterize the trade-off between power consumption at the transmitter and achieved throughput as a function of the variation over the subset size $N_{R F}$.

As already observed in the SER results, performance trends for both 4-PSK and 8-PSK are preserved. More specifically, the proposed algorithms are all able to greatly outperform schemes from the literature for all the spectrum of $N_{R F}$ values. At the same time, the proposed schemes achieve their maximum power efficiency between $N_{R F}=6$ and $N_{R F}=8$ for both 4-PSK and 8-PSK. This shows that systems with low numbers of active antennas can provide reasonable performance with a very positive trade-off between hardware complexity and power consumptions (i.e., when compared to the simplified chosen scenario where $N_{R F}=K$ ). For a direct performancecomplexity comparison between the schemes, we collect in Table I, the computational burdens required per frame to achieve the optimal value of power efficiency shown in Fig.9. There are evident complexity savings achieved by the heuristic schemes compared to the MIP approach, with little loss on the maximum power efficiency. On the other hand their complexity is comparable to conventional CapMax, with a more than $50 \%$ power efficiency improvement and a $\sim 94 \%$ reduction in the RF chains required to achieve maximum PE.

\section{Robustness to CSI}

In order to characterize the performances of the proposed CSI-robust scheme, we introduce a conventional robust scheme from the literature, which will be used
TABLE I

COMPutational BuRdens For Optimal Power EfFiciency POINTS

\begin{tabular}{|l|c|c|l|}
\hline Name & Max $\eta_{T}$ & Time $[s]$ & $N_{R F}$ \\
\hline 1-step MFCAS & 24.78 & 0.44 & 7 \\
\hline 2-step MFCAS-CBF & 26.83 & 0.85 & 6 \\
\hline 3-step CBF-CAS-CBF & 27.59 & 1.31 & 6 \\
\hline MIP-CASP & 28.38 & 2 & 5 \\
\hline SINR-CapMax & 18.4 & 0.55 & 10 \\
\hline
\end{tabular}

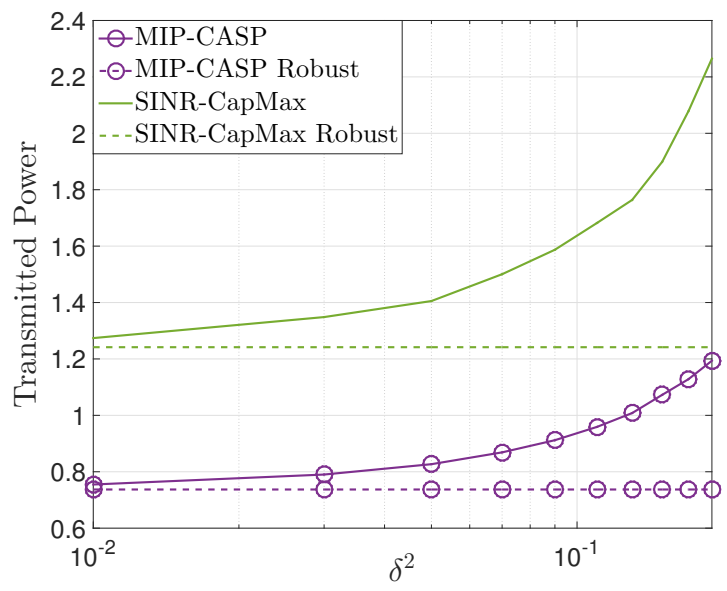

Fig. 11. Transmitted power for 4-PSK transmission when $N=100$, $K=5$ and $N_{R F}=5$.

as a benchmark technique. In line with the previous approaches, when considering the benchmark scheme we assume a cascade of TAS, based on capacity, followed by a SINR metric-based precoding. More specifically, when considering robust precoding, it is common to proceed in identifying an optimization problem that aims to minimize the transmitted power required to overcome the worst-case scenario. Accordingly, the conventional robust precoding can be defined as follows [53]

$$
\begin{aligned}
& \mathcal{P}_{10}: \underset{\mathbf{P}_{k} \geq \mathbf{0}, s_{k} \geq 0}{\operatorname{minimize}} \sum_{k=1}^{K} \operatorname{tr}\left[\mathbf{P}_{k}\right] \\
& \text { subject to }\left[\begin{array}{cc}
D_{k} & \hat{\mathbf{h}}_{k}^{T} \mathbf{Q}_{k} \\
\mathbf{Q}_{k} \hat{\mathbf{h}}_{k}^{*} & \mathbf{Q}_{k}+s_{k} \mathbf{I}_{N}
\end{array}\right] \geq \mathbf{0} \\
& D_{k}=\hat{\mathbf{h}}_{k}^{T} \mathbf{Q}_{k} \hat{\mathbf{h}}_{k}^{*}-\gamma_{k} N_{0}-s_{k} \delta_{k}^{2} \\
& \mathbf{Q}_{k}=\mathbf{p}_{k} \mathbf{p}_{k}^{H}-\gamma_{k} \sum_{i=1, i \neq k}^{K} \mathbf{p}_{i} \mathbf{p}_{i}^{H}
\end{aligned}
$$

where the notation $\mathbf{A} \geq \mathbf{0}$ is used to impose that the matrix $\mathbf{A}$ is semidefinite positive.

In Fig.11 we compare the two robust schemes in terms of transmitted power as a function of $\delta_{k}=\delta, \forall k$. In addition to the robust schemes, the minimum transmitted power for non-robust approaches is also presented. The proposed scheme is characterized by significantly lower requirements in terms of transmitted power, when compared to both robust and non-robust approaches from 
the literature. Additionally, SINR-CapMax Robust scheme from the literature is affected by a faster growth rate when compared to MIP-CASP Robust.

\section{Conclusions}

In this paper, we prove that antenna selection and precoding based on constructive multiuser interference concepts can be jointly used to greatly improve the power efficiency of future M-MIMO systems. We showed through analytical and numerical studies that constructive interference at the receiver side can be optimized by simultaneously identifying a subset of transmitting antennas and the precoded signal at the base station. We characterized the presented schemes by analyzing the computational costs in terms of running time and compared it with state-of-the-art algorithms. We evaluated performances in terms of symbol error rate, sum rate and power efficiency to analyze the performance-complexity trade-offs introduced by the proposed scheme. Our analysis and results have shown that the proposed approaches offer a favorable performance-complexity trade-off compared to conventional approaches, with a close-to-optimal performance.

\section{REFERENCES}

[1] F. Rusek, D. Persson, B. K. Lau, E. Larsson, T. Marzetta, O. Edfors, and F. Tufvesson, "Scaling up MIMO: Opportunities and challenges with Very Large Arrays," IEEE Signal Processing Magazine, vol. 30, no. 1, pp. 40-60, January 2013.

[2] T. L. Marzetta, "Noncooperative cellular wireless with unlimited numbers of base station antennas," IEEE Transactions on Wireless Communications, vol. 9, no. 11, pp. 3590-3600, November 2010.

[3] E. Larsson, O. Edfors, F. Tufvesson, and T. L. Marzetta, "Massive MIMO for next generation wireless systems," IEEE Communications Magazine, vol. 52, no. 2, pp. 186-195, February 2014.

[4] C. Masouros, M. Sellathurai, and T. Ratnarajah, "Large-Scale MIMO transmitters in fixed physical spaces: The effect of transmit correlation and mutual coupling," IEEE Transactions on Cоттиnications, vol. 61, no. 7, pp. 2794-2804, July 2013.

[5] C. Masouros and M. Matthaiou, "Space-Constrained Massive MIMO: Hitting the wall of favorable propagation," IEEE Communications Letters, vol. 19, no. 5, pp. 771-774, May 2015.

[6] A. J. Garcia-Rodriguez and C. Masouros, "Exploiting the increasing correlation of space constrained massive MIMO for CSI relaxation," IEEE Transactions on Communications, vol. 64, no. 4, pp. 1572-1587, April 2016.

[7] S. Biswas, C. Masouros, and T. Ratnarajah, "Performance analysis of large multiuser MIMO systems with space-constrained 2-D antenna arrays," IEEE Transactions on Wireless Communications, vol. 15, no. 5, pp. 3492-3505, May 2016.

[8] A. Molisch and M. Win, "MIMO Systems with antenna selection," IEEE Microwave Magazine, vol. 5, pp. 46-56, March 2004.

[9] S. Sanayei and A. Nosratinia, "Antenna selection in MIMO systems," IEEE Communications Magazine, vol. 42, no. 10, pp. 68-73, October 2004.

[10] A. Gorokhov, D. Gore, and A. Paulraj, "Receive antenna selection for MIMO spatial multiplexing: Theory and algorithms," IEEE Transactions on Signal Processing, vol. 51, no. 11, pp. 2796-2807, November 2003.

[11] Z. Chen, J. Yuan, B. Vucetic, and Z. Zhou, "Performance of Alamouti scheme with transmit antenna selection," Electronics Letters, vol. 39, no. 23, pp. 1666-1668, November 2003.
[12] R. Heath and A. Paulraj, "Antenna selection for spatial multiplexing systems based on minimum error rate," IEEE International Conference on Communications (ICC), vol. 7, pp. 2276-2280, 2001.

[13] T. Gucluoglu and T. Duman, "Performance analysis of transmit and receive antenna selection over flat fading channels," Wireless Communications, IEEE Transactions on, vol. 7, no. 8, pp. 30563065, August 2008.

[14] A. Molisch, M. Win, and J. Winters, "Capacity of MIMO systems with antenna selection," IEEE International Conference on Communications, vol. 2, pp. 570-574, 2001.

[15] R. Chen, R. W. Heath, and J. G. Andrews, "Transmit selection diversity for unitary precoded multiuser spatial multiplexing systems with linear receivers," IEEE Transactions on Signal Processing, vol. 55, no. 3, pp. 1159-1171, March 2007.

[16] P. V. Amadori and C. Masouros, "Interference-Driven antenna selection for massive multiuser MIMO," IEEE Transactions on Vehicular Technology, vol. 65, no. 8, pp. 5944-5958, August 2016.

[17] K. Dong, N. Prasad, X. Wang, and S. Zhu, "Adaptive antenna selection and Tx/Rx beamforming for large-scale MIMO systems in 60 $\mathrm{GHz}$ channels," EURASIP Journal on Wireless Communications, vol. 2011, no. 59, August 2011.

[18] B. Tae-Won and J. Bang Chul, "A practical antenna selection technique in multiuser massive MIMO networks," IEICE Transactions on Communications, vol. 96, no. 11, pp. 2901-2905, 2013.

[19] X. Gao, O. Edfors, F. Tufvesson, and E. G. Larsson, "Massive MIMO in real propagation environments: Do all antennas contribute equally?" IEEE Transactions on Communications, vol. 63, no. 11, pp. 3917-3928, November 2015.

[20] H. Li, L. Song, and M. Debbah, "Energy efficiency of large-scale multiple antenna systems with transmit antenna selection," IEEE Transactions on Communications, vol. 62, no. 2, pp. 638-647, February 2014.

[21] B. M. Lee, J. Choi, J. Bang, and B.-C. Kang, "An energy efficient antenna selection for large scale green MIMO systems," IEEE International Symposium on Circuits and Systems (ISCAS), pp. 950-953, May 2013.

[22] S. Mahboob, R. Ruby, and V. Leung, "Transmit antenna selection for downlink transmission in a massively distributed antenna system using convex optimization," International Conference on Broadband, Wireless Computing, Communication and Applications (BWCCA), pp. 228-233, November 2012.

[23] A. J. Garcia-Rodriguez, C. Masouros, and P. Rulikowski, "Reduced switching connectivity for large scale antenna selection," IEEE Transactions on Communications, vol. PP, no. 99, pp. 1-1, 2017.

[24] P. V. Amadori and C. Masouros, "Low RF-complexity millimeterwave beamspace-MIMO systems by beam selection," IEEE Transactions on Communications, vol. 63, no. 6, pp. 2212-2223, June 2015.

[25] J. Brady, N. Behdad, and A. M. Sayeed, "Beamspace MIMO for millimeter-wave communications: System architecture, modeling, analysis, and measurements," IEEE Transactions on Antennas and Propagation, vol. 61, no. 7, pp. 3814-3827, July 2013.

[26] A. Alkhateeb, G. Leus, and R. W. Heath, "Limited feedback hybrid precoding for multi-user millimeter wave systems," IEEE transactions on wireless communications, vol. 14, no. 11, pp. 6481-6494, November 2015.

[27] A. Garcia-Rodriguez, V. Venkateswaran, P. Rulikowski, and C. Masouros, "Hybrid Analog-Digital Precoding revisited under realistic RF modeling," IEEE Wireless Communications Letters, vol. 5, no. 5, pp. 528-531, October 2016.

[28] C. Masouros, "Correlation rotation linear precoding for MIMO broadcast communications," IEEE Transactions on Signal Processing, vol. 59, no. 1, pp. 252-262, January 2011.

[29] C. Masouros and G. Zheng, "Exploiting known interference as green signal power for downlink beamforming optimization," IEEE Transactions on Signal Processing, vol. 63, no. 14, pp. 3628-3640, July 2015.

[30] F. A. Khan, C. Masouros, and T. Ratnarajah, "Interference-driven linear precoding in multiuser MISO downlink cognitive radio network," IEEE Transactions on Vehicular Technology, vol. 61, no. 6, pp. 2531-2543, July 2012. 
[31] C. Masouros, T. Ratnarajah, M. Sellathurai, C. Papadias, and A. Shukla, "Known interference in the cellular downlink: A performance limiting factor or a source of green signal power?" IEEE Communications Magazine, vol. 51, no. 10, pp. 162-171, October 2013.

[32] G. Zheng, I. Krikidis, C. Masouros, S. Timotheou, D. A. Toumpakaris, and Z. Ding, "Rethinking the role of interference in wireless networks," IEEE Communications Magazine, vol. 52, no. 11, pp. 152-158, November 2014.

[33] M. Alodeh, S. Chatzinotas, and B. Ottersten, "Constructive multiuser interference in symbol level precoding for the MISO Downlink channel," IEEE Transactions on Signal Processing, vol. 63, no. 9, pp. 2239-2252, May 2015.

[34] _ , "Energy-Efficient symbol-level precoding in Multiuser MISO based on relaxed detection region," IEEE Transactions on Wireless Communications, vol. 15, no. 5, pp. 3755-3767, May 2016.

[35] P. V. Amadori and C. Masouros, "Constant Envelope Precoding by interference exploitation in Phase Shift Keying-Modulated multiuser transmission," IEEE Transactions on Wireless Communications, vol. 16, no. 1, pp. 538-550, January 2017.

[36] C. Masouros and E. Alsusa, "Dynamic linear precoding for the exploitation of known interference in MIMO broadcast systems," IEEE Transactions on Wireless Communications, vol. 8, no. 3, pp. 1396-1404, March 2009.

[37] _ - "Soft linear precoding for the downlink of DS/CDMA communication systems," IEEE Transactions on Vehicular Technology, vol. 59, no. 1, pp. 203-215, January 2010.

[38] A. Kalantari, M. Soltanalian, S. Maleki, S. Chatzinotas, and B. Ottersten, "Directional modulation via symbol-level precoding: A way to enhance security," IEEE Journal of Selected Topics in Signal Processing, vol. 10, no. 8, pp. 1478-1493, December 2016.

[39] M. P. Daly and J. T. Bernhard, "Directional modulation technique for phased arrays," IEEE Transactions on Antennas and Propagation, vol. 57, no. 9, September 2009.

[40] M. T. Kabir, M. R. Khandaker, and C. Masouros, "Interference exploitation in full duplex communications: Trading interference power for both uplink and downlink power savings," arXiv preprint arXiv:1703.10666, 2017.

[41] A. Li and C. Masouros, "Exploiting constructive mutual coupling in p2p mimo by analog-digital phase alignment," IEEE Transactions on Wireless Communications, vol. 16, no. 3, pp. 1948-1962, 2017.

[42] D. Kwon, H. S. Kang, and D. K. Kim, "Robust interference exploitation-based precoding scheme with quantized CSIT," IEEE Communications Letters, vol. 20, no. 4, pp. 780-783, April 2016.

[43] M. Alodeh, S. Chatzinotas, and B. Ottersten, "Symbol-Level Multiuser MISO Precoding for Multi-level Adaptive Modulation: A Multicast View," arXiv:1601.02788., 2016.

[44] H. Yang and T. L. Marzetta, "Performance of conjugate and Zero-Forcing beamforming in Large-Scale antenna systems," IEEE Journal on Selected Areas in Communications, vol. 31, no. 2, pp. 172-179, February 2013.

[45] M. Joham, W. Utschick, and J. Nossek, "Linear transmit processing in MIMO communications systems," IEEE Transactions on Signal Processing, vol. 53, no. 8, pp. 2700-2712, August 2005.

[46] S. Mohammed and E. Larsson, "Per-Antenna constant envelope precoding for large Multi-User MIMO systems," IEEE Transactions on Communications, vol. 61, no. 3, pp. 1059-1071, March 2013.

[47] M. Schubert and H. Boche, "Solution of the multiuser downlink beamforming problem with individual SINR constraints," IEEE Transactions on Vehicular Technology, vol. 53, no. 1, pp. 18-28, January 2004.

[48] A. B. Gershman, N. D. Sidiropoulos, S. Shahbazpanahi, M. Bengtsson, and B. Ottersten, "Convex optimization-based beamforming," IEEE Signal Processing Magazine, vol. 27, no. 3, pp. 62-75, May 2010.

[49] S. Boyd and L. Vandenberghe, Convex Optimization. Cambridge University Press, 2010.

[50] A. Garcia-Rodriguez, C. Masouros, and L. Hanzo, "Pre-Scaling optimization for Space Shift Keying based on Semidefinite Relax- ation," IEEE Transactions on Communications, vol. 63, no. 11, pp. 4231-4243, November 2015.

[51] Z. Chen, J. Yuan, and B. Vucetic, "Analysis of transmit antenna selection/maximal-ratio combining in Rayleigh fading channels," IEEE Transactions on Vehicular Technology, vol. 54, no. 4, pp. $1312-1321$, July 2005.

[52] T. L. Marzetta, "How much training is required for multiuser MIMO?" Asilomar Conference on Signals, Systems and Computers (ACSSC), pp. 359-363, October 2006.

[53] G. Zheng, K.-K. Wong, and T.-S. Ng, "Robust linear MIMO in the Downlink: A worst-case optimization with ellipsoidal uncertainty regions," EURASIP Journal on Advances in Signal Processing, 2008.

[54] D. Ha, K. Lee, and J. Kang, "Energy efficiency analysis with circuit power consumption in massive MIMO systems," IEEE 24th International Symposium on Personal Indoor and Mobile Radio Communications (PIMRC), pp. 938-942, September 2013.

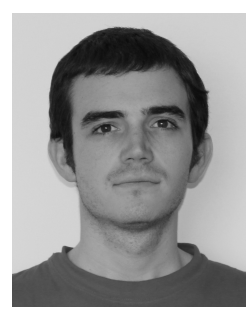

Pierluigi Vito Amadori (S'14), received the M.Sc. degree with honours in telecommunications engineering from the University of Rome La Sapienza, Rome, Italy, in 2013. Between 2012 and 2013 he held a JPL Visiting Student Researchers Program position in the Jet Propulsion Laboratory Pasadena, CA (USA). $\mathrm{He}$ is currently pursuing the Ph.D. degree in the Department of Electrical \& Electronic Engineering at University College London, London, U.K. His main research interests include wireless communications with emphasis on large antenna array systems and energy efficient communications.

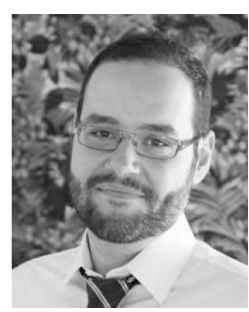

Christos Masouros (M'06, SM'14), is currently a Senior Lecturer in the Dept. of Electrical \& Electronic Eng., University College London. He received his Diploma in Electrical \& Computer Engineering from the University of Patras, Greece, in 2004, MSc by research and $\mathrm{PhD}$ in Electrical \& Electronic Engineering from the University of Manchester, UK in 2006 and 2009 respectively. He has previously held a Research Associate position in University of Manchester, UK and a Research Fellow position in Queen's University Belfast, UK. He has held a Royal Academy of Engineering Research Fellowship 2011-2016.

His research interests lie in the field of wireless communications and signal processing with particular focus on Green Communications, Cognitive Radio, interference mitigation techniques for MIMO and he is the principal investigator of the EPSRC project EP/M014150/1 on Large Scale Antenna Systems. He was the recipient of the Best Paper Award in the IEEE GlobeCom conference 2015, and has been recognised as an Exemplary Reviewer for the IEEE Transactions on Communications. $\mathrm{He}$ is an Associate Editor for IEEE Communications Letters, an Editor for IEEE Transactions on Communications, and is or has been a Guest Editor for IEEE Journal on Selected Topics in Signal Processing issue "Hybrid Analog - Digital Signal Processing for Hardware-Efficient Large Scale Antenna Arrays" and "Exploiting Interference towards Energy Efficient and Secure Wireless Communications". 